\title{
EPISODIC RETINAL ISCHAEMIA CAUSED BY REDUCED CEREBRAL VASCULAR RESISTANCE ("BRAIN STEAL")*†
}

\author{
BY \\ SIMON BEHRMAN \\ London
}

Hutchinson (1892) described an unusual syndrome of "liability to temporary total blindness on assuming the erect position". The patient who had consulted him "about arthritis" vouchsafed "incidentally" the information that she was "liable to lose her sight". Hutchinson pressed for further details, and recorded these as follows:

"Suddenly rising to the erect position, would place her for a short time in total darkness. She thought that the darkness lasted usually 'as long perhaps as you could count thirty'. On two occasions, when holding a candle, she should, she said, have set fire to objects near her had not her friends prevented it. She was accustomed to carve at table, and several times on rising to do so had been obliged to wait for a minute or so because of total darkness. Her sight always returned in a very short time, and without any headache, or other inconvenience".

Bearing in mind the dramatic and unnerving nature of sudden bilateral blindness, albeit temporary, it is surely noteworthy that the patient neither mentioned this symptom initially, nor gave any indication subsequently how long it had been troubling her. One must infer that she made light of this symptom because it was of long standing, and she had learned to live with it. A search of the literature has failed to disclose a comparable case, but the following patient would appear to duplicate this syndrome.

\section{Case Report}

An athletic man aged 24 is in normal health apart from a liability to brief attacks of bilateral blindness provoked by rising into the erect position, either from the recumbent or sitting attitudes. He became subject to these attacks in his early teens. The facility with which this blacking-out of vision can be precipitated seems to vary from time to time, but the liability to this disturbance never disappears entirely. He has never experienced any postural dizziness, tinnitus, or fainting, either concurrently with amaurosis or at any other time. He can remain in the erect position without ever developing any symptoms suggestive of postural arterial hypotension. Stimulation of carotid sinuses does not give rise to any abnormal symptoms. Carotid angiography some years ago revealed normal filling and cross circulation. Examination of the central nervous system including ophthalmoscopy is also negative. Blood pressure readings in the recumbent and standing positions were within normal limits. 
A striking feature in this case is that, of the many dozens of attacks of blacking-out of vision he has experienced in the course of a decade or so, all except two were substantially of the same duration, viz. "time required to count to thirty". These two attacks were exceptional because of the time taken for vision to return. After one such episode, he was left with a permanent inferior altitudinal hemianopia of the right eye of the type occasionally seen after a retinal ischaemic episode (Priestley Smith, 1884; Bedell, 1937). In one of his subsequent attacks, provoked in the usual manner, some 90 minutes elapsed before normal vision returned to the left eye.

\section{Discussion}

As a preliminary to the analysis of the possible mechanism underlying this syndrome, reference must be made to certain physiological facts:

(1) Normal intra-ocular pressure is in the region of $20 \mathrm{~mm}$. Hg.

(2) The brain cerebral blood flow remains remarkably constant in a variety of physiological states, and under conditions of a "steady state" is unaffected by variations of either arterial or intracranial pressures. It is evident that, for the cerebral blood to remain constant under these varying conditions, the cerebral circulation must possess means of autoregulation.

It is now generally agreed that this autoregulation is effected by two main mechanisms:

(1) Alteration of brain vascular bed by variations in the $\mathrm{CO}_{2}$ concentration.-Though the dimensions of the cerebral vascular bed are largely determined by $\mathrm{CO}_{2}$ tension, this control is not instantaneous as was shown by Rapela and Green (1964).

By cannulation at the point of confluence of the sagittal and straight sinuses, and after ligaturing the lateral sinuses, these authors measured the venous outflow from the portion of the canine brain drained by these sinuses. The reduction of cerebral arterial pressure from 120 to $50 \mathrm{~mm}$. $\mathrm{Hg}$ for a period of one minute resulted immediately in a fall of cerebral venous outflow $(\mathrm{ml} . / \mathrm{min}$.) by some 50 per cent. The cerebral outflow then gradually rose again returning to the initial level. Thus, despite a 60 per cent. reduction of cerebral arterial pressure, a normal rate of cerebral blood flow was re-established within an interval of 30 seconds. When, after one minute, the cerebral arterial pressure was restored to normal, the cerebral venous outflow immediately increased by some 75 per cent. It then fell, returning to the previous level within about 30 seconds, when cerebral vascular resistance could be assumed to have recovered its normal magnitude (Figure, opposite).

The effect of this particular experiment was to increase the rate of cerebral venous outflow, for a very short period, by 75 per cent. In terms of human cerebral blood flow, such an increment would represent a rise from 800 to $1,400 \mathrm{ml} . / \mathrm{min}$. and this illustrates the magnitude of fluctuations of cerebral vascular resistance. Under extreme conditions, as following experimental acute arrest of cerebral circulation in man (by raising the pressure to $600 \mathrm{~mm}$. $\mathrm{Hg}$ in a cervical pressure cuff), the cerebral circulation may overshoot to such a degree as to lead to transient cerebral acapnia so that immediately after full recovery of consciousness, after this experiment, a seizure may occur (Rossen, Kabat, and Anderson, 1943).

(2) By contraction of smooth muscle cells of the arterioles in response to an increased pressure across the vascular wall.-This has been demonstrated by direct measurements of pial arterioles, and indirect evidence of this control is also provided by the 


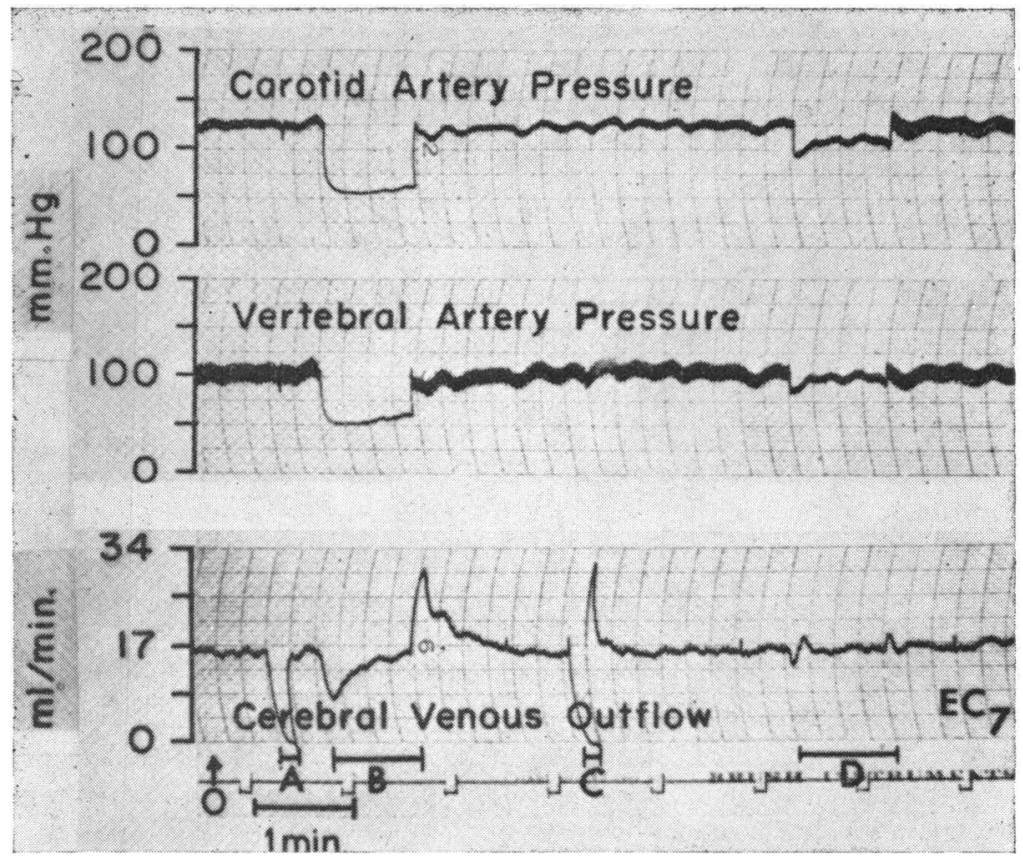

FIGURE.-Carotid and vertebral artery pressure $(\mathrm{mm} . \mathbf{H g}$ ) and cerebral venous outflow (ml./min.). Reduction of carotid and vertebral arterial pressure to 50 $\mathrm{mm}$. Hg for 1 minute is seen at "B". (From Rapela and Green, 1964, p. 205, by permission of the Editor of Circulation Research.)

immediate rise of central retinal artery pressure on compression of the jugular veins (Berens, Smith, and Cornwall, 1928). Under normal conditions, variation of central retinal artery pressure provides a reliable index of the corresponding changes in intracarotid pressure.

\section{Effect on Retinal Circulation of Rising from the sitting into the ERECT Position}

On rising there is an immediate drop in venous pressure, and also, therefore, in intracranial venous pressure, and for a short period before the normal feed-back control of the cerebral vascular bed can come into effect cerebral vascular resistance is low. Cerebral blood flow is therefore greatly increased until the small cerebral blood vessels assume the diameter appropriate to the new conditions. This transient state of diminished vascular resistance of the brain must result in a temporary fall of the intracarotid pressure.

Information on pressure changes in the carotid under these conditions is lacking, but some relevant data are provided by intra-arterial blood pressure measurements in man, while subjected to tipping from the horizontal to the head-up position (Lomas, Dameshek, Myerson, and Goldman, 1936).

The average fall in intracarotid pressure during this procedure was $40 \mathrm{~mm}$. $\mathrm{Hg}$ (in some cases as much as 60). Within a few seconds of reaching the upright position, there occurred a rise in pressure so that the average final fall in intracarotid pressure was $21 \mathrm{~mm} . \mathbf{H g}$. It must be borne in mind that this fall in carotid pressure was not part of a general arterial hypotension. On the contrary, immediately after the head-up tip, the observed average 
rise in brachial arterial pressure, i.e. pressure at heart level, was $20 \mathrm{~mm}$. Hg. This represents approximately the pressure required to overcome the force of gravity to raise blood from heart to head level. The fall in the carotid pressure on rising into the erect position cannot, therefore, be due to the effect of gravity.

Corresponding with the fall in carotid pressure, there is a fall in the pressure in the central retinal artery (Kamogawa, 1936). Normally this fall is too little to engender any disturbance of vision. It must be assumed, therefore, that the fall in carotid pressure in this patient is in excess of physiological limits. In the absence of any symptoms of generalized postural hypotension, this exaggeration of the physiological fall in carotid pressure might be due to stenosis of the carotid arteries in either the cervical or aortic regions.

Among 100 patients who had obstruction of the great vessels at or near their origin from the aortic arch, some are known to have complained of dimness of vision on rising rapidly from the sitting position (Ross and McKusick, 1953). In the case reported here there was neither angiographic evidence of abnormality of the carotids in the cervical and cranial regions, nor was there any clinical evidence of the "aortic arch syndrome". A cerebral vascular malformation can also be excluded on the basis of the angiography.

It must be concluded that, under conditions of greatly reduced cerebral vascular resistance prevailing immediately on rising into the erect position, the fall in carotid pressure, and also therefore in the central retinal artery is, in this case, of such magnitude that the final pressure does not sufficiently exceed the intra-ocular pressure to maintain retinal circulation. Such a haemodynamic situation might arise if the pressure equalization afforded by the circle of Willis were lacking. A congenital absence of both posterior communicating arteries might explain the incompetence of the circle of Willis.

From the experiments of Rapela and Green just quoted, it can be inferred that within a period of 15-30 seconds, appropriate cerebral vascular adjustments take place, with consequent restoration of normal retinal circulation. The habitual return of vision within 15-30 seconds supports this conclusion. It can also be assumed that, if adequate retinal circulation is not restored within a critical period, vision may either remain in abeyance for an unduly long period, or may be irretrievably lost. As was mentioned, both these phenomena have occurred in this case.

\section{Monocular Amaurosis Fugax}

Both in respect of duration and in some other clinical characteristics, the amaurotic phenomena in this case resemble the evanescent, stereotyped attacks of monocular amaurosis encountered in occlusive disease of the carotid (Behrman, 1954). This would suggest that the immediate causative factor is the same, viz. a brief, self-terminating period of reduced brain vascular resistance causing alteration in the distribution of carotid flow in response to otherwise symptomless intracranial hypotension. Such hypotension might arise, for instance, when, given an appropriate overall situation, the head is turned into a position in which blood flow in one of the patent transcervical arteries ceases. The consequent abrupt fall of cerebral vascular resistance, before the "steady state" becomes re-established, might lead to brief blacking-out of the eye on the side on which, because of carotid occlusion, the ophthalmic artery obtains its blood supply by way of the circle of Willis (de Kleyn, 1939; Toole and Tucker, 1960).

"Brain steal" might be an appropriate term to describe this haemodynamic situation in which, as a result of lowering of cerebral vascular resistance, the diversion of blood to the brain causes the pressure within the internal carotid artery to fall below that of the intraocular pressure. 
"Brain Steal" due to Cerebral haemangioma

The following case illustrates the phenomenon of "brain steal" caused by reduced cerebral vascular resistance as a result of an arterio-venous malformation.

A girl aged 7 was in excellent health until she began to complain of mild headache in the morning. A sudden exacerbation of the headache, accompanied by vomiting, occurred later in the day. On admission to hospital on the third day of her illness, she was semi-conscious, and had neck stiffness, and blood was found in the cerebrospinal fluid. A few hours later she had several generalized seizures following which she developed left hemiparesis. Carotid angiography revealed a vascular malformation, the main feeding vessel of which was the right middle cerebral artery. After 30 ml. "old" fluid blood had been aspirated from the right occipital lobe in which the angioma was situated, the child's condition gradually improved, and it was then recognized that the left eye was totally blind, and that a nasal hemianopia was present in the right field of vision. About 4 months after the onset of symptoms, the outer two-thirds of the right occipital lobe containing the entire vascular malformation was excised by Mr. J. R. Gibbs. Check angiography later confirmed that a total excision of the arterio-venous angioma had been effected. She was free from symptoms when seen 3 years later, but the visual defects have remained unchanged.

The abrupt onset of blindness in the left eye evidently occurred at the time of the ictus when, because of the subarachnoid bleeding, carotid spasm might be expected (Behrman, 1962). Under these conditions of diminished carotid blood flow, rapid shunting of much of the carotid circulation from the arterial to the venous side of the malformation might result in "brain steal" of a degree sufficient to cause irreversible retinal damage through ischaemia.

\section{Summary}

(1) A description is given of a syndrome of which the only clinical manifestation is transient bilateral retinal ischaemia on assuming the erect position.

(2) It is concluded that this retinal ischaemia is due to internal carotid hypotension without generalized postural arterial hypotension.

(3) A drop in intracranial venous pressure on assuming the erect position causes a fall in cerebral resistance of short duration. The consequent diversion of blood to the brain in individuals presenting this syndrome engenders a fall in the carotid pressure to a level below that of the intra-ocular pressure. It is suggested that this excessive fall in intracarotid pressure is due to failure of pressure equalization as a result of incompetence of circle of Willis.

(4) The amaurotic phenomena in these cases resemble those encountered in association with carotid artery occlusion. It is suggested that the latter type of uniocular amaurosis fugax is caused by a reduction in cerebral vascular resistance. Pending the re-establishment of the "steady state", the consequent fall in pressure in the ophthalmic artery, which obtains its blood supply by way of circle of Willis, may engender retinal circulatory insufficiency.

(5) "Brain steal" is put forward as a term to describe the haemodynamic situation in which, as a result of lowering of cerebral vascular resistance, the diversion of blood to the brain causes the pressure within the internal carotid artery to fall below the level of the intra-ocular pressure.

(6) A case of "brain steal" due to cerebral haemangioma is described. 
I am indebted to Mr. J. R. Gibbs, F.R.C.S., for permission to publish details of his case of cerebral haemangioma, and to the Editor of Circulation Research for permission to reproduce the Figure (Rapela and Green, 1964, p. 205).

\section{REFERENCES}

Bedell, A. J. (1937). Amer. J. Ophthal., 20, 237.

Behrman, S. (1954). Postgrad. med. J., 30, 570. (1962). Ibid., 38, 396.

Berens, C.. Smith, T. H., and Cornwall, L. H. (1928). Arch. Neurol. (Chic.), 20, 1151.

Hutchinson, J. (1892). Arch. Surg. (Lond.), 4, 184.

Kamogawa, A. (1936). Klin. Mbl. Augenheilk., 97, 611.

KleYN, A. DE (1939). Confin. neurol. (Basel), 2, 257.

Loman, J., DamesheK, W., Myerson, A., and Goldman, D. (1936). Arch. Neurol. (Chic.), 35, 1216.

Rapela, C. E., and Green, H. D. (1964). Circulat. Res., vol. 15, Supplement No. 1, p. 205.

Ross, R. S., and McKusick, V. A. (1953). Arch. intern. Med., 92, 701.

Rossen, R., Kabat, H., and Anderson, J. P. (1943). Arch. Neurol. (Chic.), 50, 510.

SMIth, E. PRIESTley (1884). Ophthal. Rev., $3,1$.

TOOLE, J. F., and TUCKER, S. H. (1960). A.M.A. Arch. Neurol., 2, 616. 